\title{
Analysis of the Impact of Misaligned Wireless Backhaul Links on Optical Attocell Networks
}

\author{
Yuhui Wu${ }^{*, 1}$, Mario Pickavet ${ }^{1}$, Didier Colle ${ }^{1}$ \\ ${ }^{*}, 1$ IDLab, Department of Information Technology, Ghent University-imec, Ghent, Belgium \\ *Tel: +32933 14977, e-mail: yuhui.wu@ugent.be
}

\begin{abstract}
Wireless solutions based on visible light communication (VLC) have been proposed for backhaul transmission in optical attocell networks. Perfect alignment of auxiliary transceivers is important for wireless backhaul links due to the requirement of direct line-of-sight (LOS) and the first-bounce specular reflection of mirror-aided non-LOS. However, the perfect alignment may not be guaranteed due to the limitation of the layout of BSs or installation mistakes. In this article, we investigate the impact of misalignment on the overall network performance. Two VLC backhaul link configurations have been considered and compared. Performance of using different frequency reuse schemes and channel allocation schemes are compared.
\end{abstract}

Keywords: visible light communication, misalignment, frequency reuse.

\section{INTRODUCTION}

Optical attocell networks based on visible light communication (VLC) have drawn scientific interests in the last few years. Many backhaul solutions for connecting VLC base stations (BSs) to the Internet have been proposed. Wired backhaul technologies, such as power-line communication (PLC) and power-over-Ethernet (PoE), have been proposed as the backhaul solution. However, wired backhaul solutions require installing additional control devices like modems or replacing current power cables. The cost for installation and maintenance could be extremely high. Wireless backhaul solutions based on VLC links are proposed in [1], [2]. VLC backhaul links are viable solutions for optical attocell networks which have used VLC for access data transmission. One drawback of using VLC backhaul links is the impact caused by misaligned auxiliary transceivers, as misalignment violets the strict line-of-sight (LOS) condition of VLC backhaul links. In many indoor scenarios, perfect alignment may not be guaranteed due to the limitation of the layout of BSs or installation mistakes. Thus, it is necessary to investigate the misalignment impact on optical attocell networks. Three frequency reuse schemes are proposed. Static subcarrier allocation based on the frequency reuse scheme is considered. Simulation results of networks using two backhaul link configurations are presented and compared.

\section{SYSTEM MODEL}

This section introduces VLC-based optical attocell networks considered in our work. Different link configurations and channel gain calculations are explained. Three frequency reused schemes: fully reused (FR), in-band (IB) mode 1, and IB mode 2. Allocation of subcarriers of optical orthogonal frequency division multiplexing (OFDM) is based on the chosen frequency reused scheme.

\subsection{Visible Light Communication}
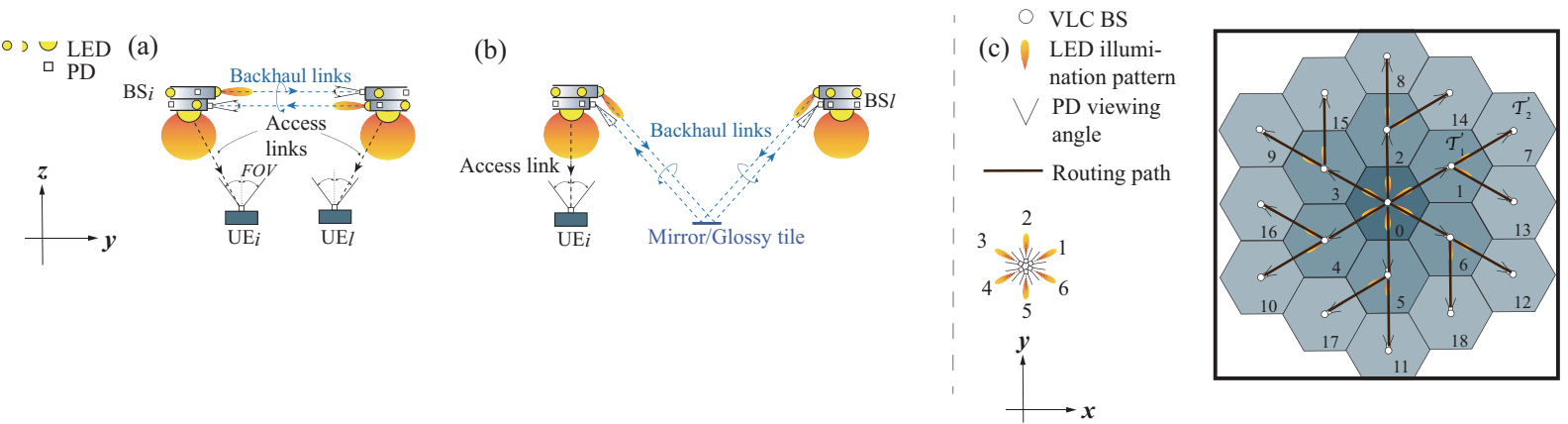

Figure 1: (a) LOS link configuration; (b) Mirror-aided non-LOS link configuration; (c) Two-tier optical attocell network with central gateway

A VLC communication system uses a visible light-emitting-diode (LED) to send intensity modulated (IM) signal which is high-frequency modulated on the light intensity. A photodiode (PD) receiver converts the received light to electrical current and decodes the received signal. Optical OFDM, such as direct-current optical OFDM (DCOOFDM), is commonly used for VLC system due to its spectral efficiency. As IM modulated signal must be real, 
the 0 -th and $(K / 2)$-th subcarriers are set to zero, where the $(K / 2)$-th subcarrier is the Nyquist term, and $K$ is the number of OFDM subcarriers. Transmitted electrical power $P_{\text {elec }}$ is equally distributed among $K-2$ datacarrying subcarriers, i.e., $P_{\text {elec }, k}=P_{\text {elec }} /(K-2)$. In order to ensure non-negative optical intensity, DCO-OFDM adds DC-bias to the modulated signal and clips all negative values. The clipping noise can be neglected when choosing an appropriate biased factor $a$ and we use $a=3$ in following calculation. The transmitted electrical power $P_{\text {elec }}$ can be calculated based on the average transmitted optical power $P_{o p t}$ and the bias factor $a$ based on the relationship $a=P_{\text {opt }} / \sqrt{P_{\text {elec }}}$. The optical power $P_{\text {opt }}$ is the mean value of the time-domain signal after adding the DC bias [3]. Transmitted optical power $P_{\text {opt }}$ is limited by illumination and eye safety requirements, which will be discussed in section 3.1.

According orientation of the communicating LED-PD pair, VLC links are categorized into line-of-sight (LOS) and non-LOS link configurations. In [2], mirror-aided non-LOS configuration is introduced, which improve the quality of non-LOS links by adding first-bounce specular reflection. LOS and mirror-aided non-LOS configurations are illustrated in Fig. 1(a) and (b), respectively. As LED lamps are Lambertian light sources, the received power of a LOS link can be calculated by Lambertian emission law. The LOS channel gain, which is the ratio of received power and transmitted power, is obtained by following equation[4]

$$
G_{s, r}=\frac{(m+1) A_{r}}{2 \pi D_{s, r}^{2}} \cos ^{m}(\phi) \cos (\theta) \operatorname{rect}\left(\frac{\theta}{\Theta}\right),
$$

where $\phi$ and $\theta$ are angles of LOS paths measured from orientations of a sender $s$ and a receiver $r$, respectively, $D_{s, r}$ is the Euclidean distance from $s$ to $r, A_{r}$ is the active area of the PD, $\Theta$ is the field-of-view (FOV) of the PD. A Lambert order $m=-\ln 2 / \ln \left(\cos \left(\Phi_{1 / 2}\right)\right)$ is used to describe LED's emission pattern, where $\Phi_{1 / 2}$ is the semi-angle at half-intensity of the LED emission pattern. The rectangular function $\operatorname{rect}(x)$ is 1 if $|x| \leq 1$, and 0 otherwise. Although LOS component is missed in mirror-aided non-LOS configuration, this configuration takes advantage of the first-bounce specular reflection component. The mirror-aided non-LOS path can be seen as a "mirror-obstructed" LOS path[2]. The mirror-aided non-LOS channel gain can be calculated using equation (1) as the LOS channel. The gain of a mirror-aided non-LOS channel from a sender $s$ to a receiver $r$ is $\rho_{m} G_{s^{\prime}, r}$, where $s^{\prime}$ is the mirror image of $s$ and $\rho_{m}$ is the reflectivity of the mirror[2].

\subsection{Optical Attocell Network}

A VLC-based optical attocell network uses LED lamps as BSs. Each BS covers an attocell with radius ranging from one to several meters. User equipment (UE) communicate with the nearest BS via VLC downlink and infrared (IR) uplink. A gateway, which can be one of the VLC BSs, connect the optical attocell network to the Internet. In this paper, we consider a two-tier ring type optical attocell network as shown in Fig. 1(c). The gateway is the central BS denoted as BS0. Other BSs are categorized into tier-1 BSs $\mathcal{T}_{1}$ and tier-2 BSs $\mathcal{T}_{2}$. Fixed routing is used in our work as shown in the figure. When the gateway receives data with the destination in a tier-2 cell (cell 7 to 18), it forwards this downlink information by using one of its neighboring tier-1 BSs (BS1 to BS6) as relay. Uplink transmission in the direction from tier-2 BSs to the gateway is not considered in our work.

Communication links between BSs and the gateway form a backhaul network. VLC-based wireless backhaul network can use two different link configurations discussed in previous section. Both backhaul link configurations require auxiliary LEDs and PDs as shown in Fig. 1(a) and (b). When mirror-aided non-LOS backhaul links are used, small mirrors or small glossy floor tiles should be placed at centers of every cell edge to provide first-bounce specular reflections.

\subsection{Frequency Reuse Schemes}

As DCO-OFDM is used to modulate transmitted signal, different transmitters can use different OFDM subcarriers to send data. Receivers can convert the received signal on certain subcarriers using fast Fourier transform (FFT). Subcarriers are orthogonal to each other and Shannon-Hartley theorem can be applied individually on each subcarrier. VLC links using the same communicating subcarriers interfere with each other. The SINR on the $k$-th subcarrier of a link denoted by $l$ is calculated by

$$
\gamma_{l, k}=\eta \frac{P_{\text {elec }, l, k} R_{P D}^{2}\left|G_{l}\right|^{2}}{\sigma_{k}^{2}+\sum_{m \in \mathcal{I}_{l}} P_{\text {elec }, m, k} R_{P D}^{2}\left|G_{m}\right|^{2}},
$$

where $G_{l}$ is the channel gain of link $l$ calculated with equation (1) or the extension equation $\rho_{m} G_{s^{\prime}, r}$ introduced in section 2.1 for mirror-aided non-LOS channel, $R_{P D}$ is the responsivity of PD in A/W, $\sigma_{k}=N_{0} B / K$ is the noise power on the $k$-th subcarrier, $N_{0}$ is the power spectral density (PSD) of additive white Gaussian noise (AWGN) channels, and $B$ is the bandwidth of OFDM signal, $G_{l}$ denotes the channel gain of the link $l, P_{\text {elec }, l, k}$ 
(a)

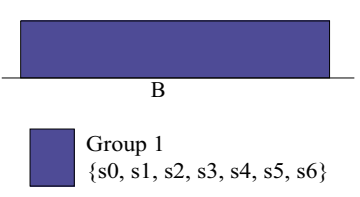

LED number

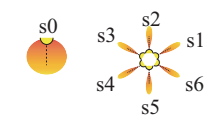

(b)

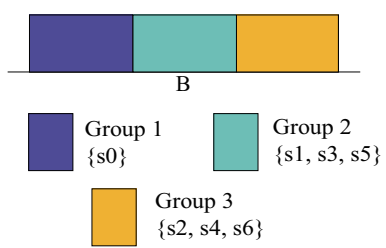

(c)

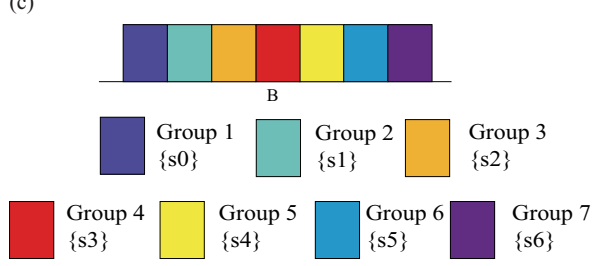

Figure 2: Illustration of spectrum allocation of frequency reuse scheme (a) FR; (b) IB mode 1; (c) IB mode 2; $B$ is the electrical bandwidth available for one BS

denotes the transmitted electrical power on the $k$-th subcarrier of link $l$. The efficiency loss factor $\eta=\frac{N-2}{N-2+N_{c p}}$ indicates the bandwidth efficiency loss caused by adding cyclic prefix (CP) at the end of each OFDM frame. Adding CP to OFDM frames can overcome inter-symbol interference (ISI) with simple equalization. We set $N_{c p}=4$ in our calculations. The interference set $\mathcal{I}_{l}$ consists of links communicating on the same subcarriers as link $l$. In this work, we assume that every BS is able to use the whole electrical spectrum. The interference set of each link is determined by the frequency reuse scheme. We describe three frequency reuse schemes based on the allocation of subcarriers.

a) Fully reuse ( $F R$ ): Every transmitter can reuse the whole spectrum. The interference set of a link from the $j$-th transmitter of a $\mathrm{BSi}$, which is denoted by $i$-s $j$, is $\mathcal{I}_{l: l(s)=i \text {-s } j}=\{m: m(s)=p$-s $n, \forall p \in[0,18], \forall n \in$ $[0,6] l(s) \neq m(s)\}$. Here, $l(s)$ denotes the originating transmitter $s$ of link $l$.

b) In-band (IB) mode 1: All downward-oriented transmitters for access data transmission use the same set of subcarriers. The rest subcarriers are divided into two groups: the first group consists of transmitters numbered with $1,3,5$, and the second group consists of transmitters numbered with 2, 4, 6. Thus, each neighboring LEDs on a single base station use different spectrum. The interference set is $\mathcal{I}_{l: l(s)=i \text {-s } j}=\{m: m(s)=p$-s $n, \forall p \in$ $[0,18], \forall n \in\{j, 1+(j+1) \bmod 6,1+(j+3) \bmod 6\}, l(s) \neq m(s)\}$. Here, $x$ mod $y$ denotes modulo operation and returns the remainder of the Euclidean division of $x$ by $y$.

c) In-band (IB) mode 2: Each transmitter on a single BS uses individual subcarrier sets to avoid inter-link interference in the same cell. The interference set is $\mathcal{I}_{l: l(s)=i \text {-s } j}=\{m: m(s)=p$-s $j, \forall p \in[0,18], p \neq m\}$.

Illustration of three frequency reuse schemes is shown in Fig. 2. The number of subcarriers used by the access group, i.e. group 1 in both Fig. 2(b) and Fig. 2(c), is determined by a bandwidth allocation ratio $\delta$. This ratio is calculated based on the requested rates of downlink traffic and will be discussed in section 3.2. The remaining $(1-\delta) K$ subcarriers are evenly assigned to each backhaul group, i.e. group 2 and 3 in Fig. 2(b) and group 2 to 7 in Fig. 2(c).

\section{NUMERIC ANALYSIS}

In order to assess the performance of networks with misaligned auxiliary transceivers using different frequency reuse schemes, we calculate the network average spectral efficiency $\bar{\rho}$ in bit/s/Hz/cell. This is the average value of the end-to-end spectral efficiency of each downlink route from the gateway to the UE. The end-to-end spectral efficiency of one downlink route is the minimal value of spectral efficiency of links along this route. If multiple routes share one link, the spectral efficiency is evenly allocated to each route. The spectral efficiency of one link is the mean of spectral efficiency on each allocated subcarrier which is calculated by $\rho_{l, k}=\delta_{l} \log _{2}\left(1+\gamma_{l, k}\right)$, where $\delta_{l}$ is the ratio of bandwidth (subcarriers) allocated to link $l$. When FR method is used, $\delta_{l}=1$ for all links. To obtain the spectral efficiency of each link, we have to determine the transmitted power and the bandwidth allocation ratio of each link.

\subsection{Illumination And Eye Safety}

Wide-beam LEDs are normally used for indoor lighting to provide uniform illumination. We assume that the semi-angle of downward-oriented LEDs $\Phi_{a}=60^{\circ}$. The primary purpose of these LEDs is illumination. Their transmitted power $P_{o p t}^{a}$ is determined by the illumination requirement. Auxiliary LEDs are used for backhaul data transmission. Increasing the transmitted power of auxiliary LEDs $P_{o p t}^{b}$ not only increases the average illuminance in the room, but also increases the risk of causing visual discomfort on human eyes. This is because that narrowbeam visible LEDs used as auxiliary LEDs emit direct and reflected light. We configure the transmitted power such that average indoor illuminance is under $750 \mathrm{~lx}$, which is the maximum value required for activities in offices. Transmitted power of auxiliary LEDs is limited such that the maximum vertical illuminance on human eyes (assuming at height of $1.5 \mathrm{~m}$ ) is $2000 \mathrm{~lx}$. Discussion of illumination requirement on transmitted power 
is presented in a separated paper. In this work, transmitted power $P_{o p t}^{a}$ and $P_{o p t}^{b}$ is calculated based on the illumination and eye safety requirements and the semi-angle of auxiliary LEDs $\Phi_{b}$. The calculated transmitted power is set to be same for both link configurations and does not change with misalignment angles.

\subsection{Bandwidth Allocation}

When IB method is used, the fraction of subcarriers used for access data transmission is determined by bandwidth allocation ratio $\delta$. As backhaul links cannot become bottleneck for data transmission to UEs, the data rate of each backhaul downlink should be large enough to provide access data transmission to all downstream UEs. We use $\mathcal{D} \mathcal{L}_{l}$ to denote the set of downstream access links of link $l$. Notice that all UEs equally share the spectrum $\delta B$ when multiple UEs are located in a cell. The sum of the data rates of these UEs achieves the maximum value when all UEs are located at a point with the maximum SINR, i.e. in the center of the cell. It is clear that the maximum sum rate is equal to the achievable data rate of a single UE located at the cell center. Thus, we assume that every BS has one UE located at its cell center which uses the whole spectrum allocated to the access link. The bandwidth allocation ratio $\delta_{l}$ of one backhaul link $l$ is

$$
\delta_{l}=\frac{\frac{1}{b} \log _{2}\left(1+\gamma_{l}\right)}{\frac{1}{b} \log _{2}\left(1+\gamma_{l}\right)+\sum_{m \in \mathcal{D} \mathcal{L}_{l}} \log _{2}\left(1+\gamma_{m}\right)},
$$

where $\gamma_{l}$ is the average SINR of link $l$ across all allocated subcarriers, $b$ is the number of spectrum groups for backhaul links, $b=2$ for IB mode 1 and $b=6$ for IB mode 2 . As a BS may have multiple backhaul links, the bandwidth allocation ratio $\delta_{B S}$ of one BS is the minimum value of $\delta_{l}$ of backhaul links of this BS. The ratio $\delta$ changes with misalignment angle as SINR of each link changes. There are two options of configuration. The first option is global configuration. All BSs are configured with the same value of $\delta$, which is the minimum value of $\delta_{B S}$ in the network. The second option configures each BS with its own calculated $\delta_{B S}$. In the first option, the minimum value of $\delta$ is obtained by comparing all calculated results in the network, while every BS only needs to calculate it's own bandwidth allocation ratio in the second option.

\section{SIMULATIONS AND RESULTS}

The performance of networks with misaligned auxiliary transceivers using different frequency reuse schemes is assessed by computer simulations. In all misalignment scenarios, we assume that all auxiliary transmitters of BS0 rotates against vertical axis with the same angle. From our simulations, we knew that misaligned BS0 may cause larger impact on the overall performance than other BSs as the gateway BS0 sends downlink information to all BSs. In real applications, configuration of $\Phi_{b}$ may depend on eye safety issues and the QoS requirement of backhaul links. Simulation results of networks using two link configurations are presented.

\subsection{Average Spectral Efficiency}

Due to the randomness of misalignment, the horizontal misalignment angle $\Delta \theta$ may vary within the range $-30^{\circ}$ to $30^{\circ}$. The negative value of angle means rotating auxiliary transceivers clock-wise. Notice that the angle of the orientation of one auxiliary transceiver with respect to its neighboring auxiliary transceiver is $60^{\circ}$. When horizontal misalignment angle exceeds $\pm 30^{\circ}$, the misaligned BS reassigns auxiliary LEDs for backhaul transmission. Although receiving PDs of tier-1 and tier-2 BSs are aligned in our simulations, we configure the FOV of receiving PDs to ensure successful backhaul data transmission in misaligned case without loss of generality. In our simulations where the height of BSs is $2.85 \mathrm{~m}$, the height of UEs is $1.15 \mathrm{~m}$, and cell radius is $2 \mathrm{~m}$, FOVs of auxiliary PDs are $30^{\circ}$ and $15.45^{\circ}$ in LOS configuration and mirror-aided non-LOS configuration, respectively. The network average spectral efficiency (ASE) $\bar{\rho}$ of two link configurations with and without misalignment is shown in Fig. 3. Due to the limit of space, we only show misalignment cases with large horizontal misalignment angle $\left(\Delta \theta=27^{\circ}\right)$. It can be seen that misalignment causes significant performance degradation for both link configurations. However, the performance of different frequency reuse schemes depends on the horizontal misalignment angle $\Delta \theta$ and semi-angle of auxiliary LEDs $\Phi_{b}$. By calculating the interferenceto-noise ratio (INR) of each link, which is the ratio between the sum of interference and the noise, we found that the performance of different frequency reuse schemes depends on the regime of the network. When the average INR of networks without frequency reuse is smaller than 1, the optical attocell network is at noise-limited regime. In this regime, using frequency reuse scheme (two IB methods) cannot provide more gain as noise is dominant. When the average INR is larger than 1, the network is at interference-limited regime. Using frequency reuse scheme could provide better SINR on some links, thereby the network performance is improved. In Fig. 3(b) we can see that there exists transition point from noise-limited regime to interference-limited regime for LOS configuration, while networks using mirror-aided non-LOS configuration are always at interference-limited regime as shown in Fig. 3(c) and (d). In addition, LOS configuration seems more vulnerable to misalignment for 


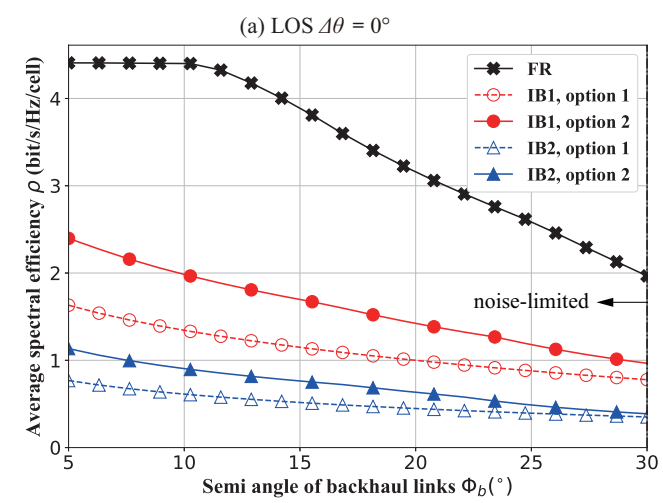

(c) Mirror-aided non-LOS $\Delta \theta=0^{\circ}$

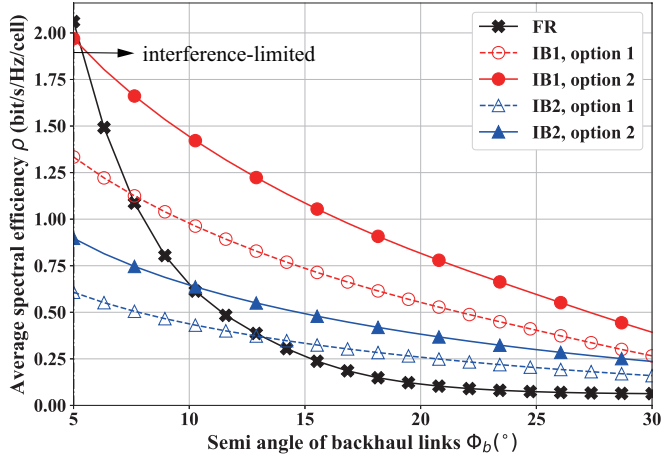

(b) $\operatorname{LOS} \Delta \theta=27^{\circ}$

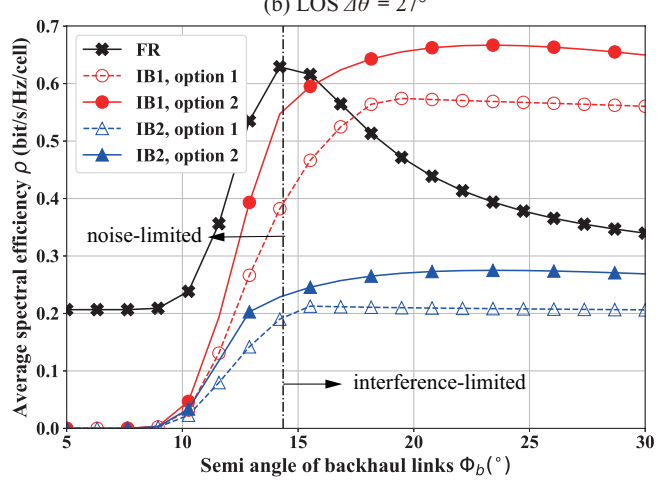

(d) Mirror-aided non-LOS $\Delta \theta=27^{\circ}$

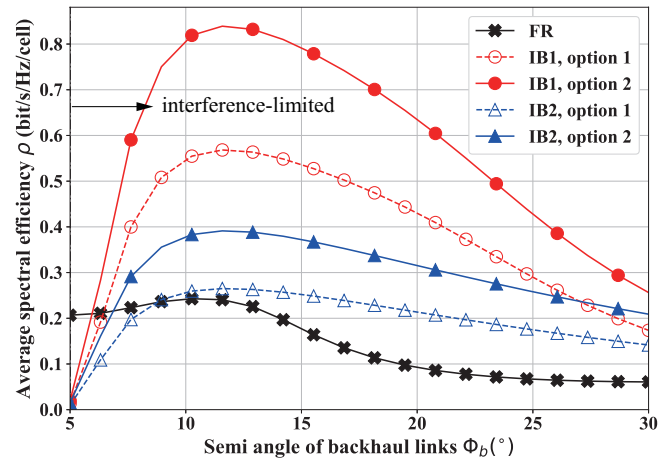

Figure 3: Average spectral efficiency of networks using (a) LOS backhaul configuration with $\Delta \theta=0^{\circ}$; (b) LOS backhaul configuration with $\Delta \theta=27^{\circ}$; (c) Mirror-aided non-LOS backhaul configuration with $\Delta \theta=0^{\circ}$; (d) Mirror-aided non-LOS backhaul configuration with $\Delta \theta=27^{\circ}$

two reasons: 1) Comparing Fig. 3(a) and (b), we can see that the maximum ASE that can be achieved by using LOS configuration significantly drops (maximum $4.4 \mathrm{bit} / \mathrm{s} / \mathrm{Hz} / \mathrm{cell}$ when aligned and maximum $0.67 \mathrm{bit} / \mathrm{s} / \mathrm{Hz} / \mathrm{cell}$ when misaligned). This is compared to mirror-aided non-LOS configuration whose maximum ASE drops from $2 \mathrm{bit} / \mathrm{s} / \mathrm{Hz} /$ cell to $0.84 \mathrm{bit} / \mathrm{s} / \mathrm{Hz} / \mathrm{cell}$ (Fig. 3(c) and (d)); 2) When the misalignment angle is large $\left(\Delta \theta=27^{\circ}\right.$ ), using LOS configuration cannot achieve more than $0.7 \mathrm{bit} / \mathrm{s} / \mathrm{Hz} /$ cell regardless the chosen semi-angle $\Phi_{b}$, while using mirror-aided non-LOS configuration is able to get more than $0.8 \mathrm{bit} / \mathrm{s} / \mathrm{Hz} / \mathrm{cell}$ when $\Phi_{b}$ is between $10^{\circ}$ to $15^{\circ}$.

\subsection{Cumulative Distribution Function of ASE}

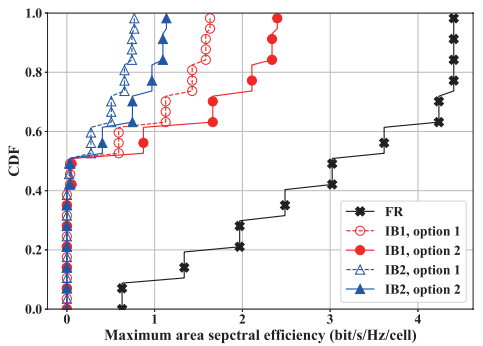

(a) LOS, noise-limited regime

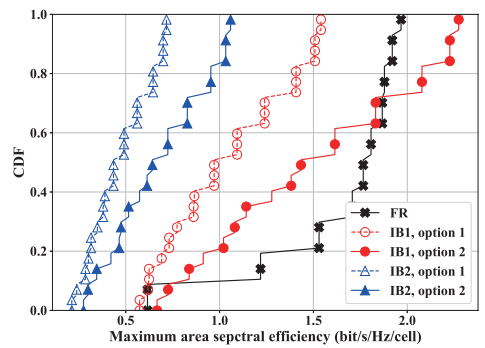

(b) LOS, interference-limited regime

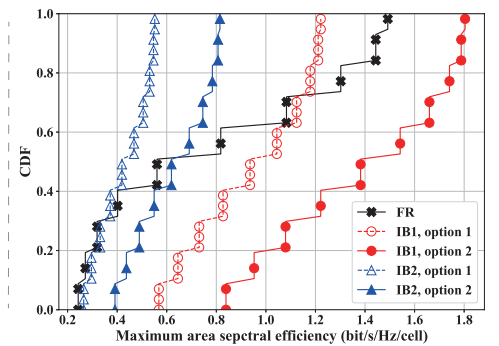

(c) Mirror-aided non-LOS, interference-limited regime

Figure 4: CDF of maximum ASE of networks using (a) LOS backhaul configuration in the noise-limited regime; (b) LOS backhaul configuration in the interference-limited regime; (c) Mirror-aided non-LOS backhaul configuration in the interference-limited regime

In order to further compare the performance of using different frequency reuse schemes, we calculate the cumulative distribution function (CDF) of maximum ASE of each frequency reuse scheme obtained by simulating two-tier optical attocell networks in different regimes. Simulation results are shown in Fig. 4. It can be seen from 
Fig. 4(a) that FR outperforms other schemes when networks using LOS configuration in the noise-limited regime. By using FR scheme, networks have more than $50 \%$ probability to get maximum ASE $\bar{\rho}_{\text {max }}>3 \mathrm{bit} / \mathrm{s} / \mathrm{Hz} / \mathrm{cell}$, while other schemes cannot provide $\bar{\rho}_{\max }$ larger than $3 \mathrm{bit} / \mathrm{s} / \mathrm{Hz} /$ cell. When networks using LOS configuration are in the interference-limited regime as shown in Fig. 4(b), IB mode 1 with option 2 can achieve better performance than FR. When networks use mirror-aided non-LOS backhaul configuration, networks are always in the interference-limited regime. Using FR method could have large probability to get poor performance as shown in Fig. 4(c). For instance, networks using IB mode 1 with option 2 always have $\bar{\rho}_{\text {max }}>0.8 \mathrm{bit} / \mathrm{s} / \mathrm{Hz} / \mathrm{cell}$, while networks using FR have near $50 \%$ probability to get $\bar{\rho}_{\text {max }}<0.8 \mathrm{bit} / \mathrm{s} / \mathrm{Hz} / \mathrm{cell}$. In addition, it is also shown that IB mode 1 with option 2 outperforms other IB methods regardless the regime and backhaul configuration. In general, FR method outperforms in the noise-limited regime, while IB mode 1 with option 2 has better performance when interference becomes larger. Given the same probability of horizontal misalignment angle, we calculated the expected values of the maximum ASE, i.e., $\mathrm{E}\left[\bar{\rho}_{\max }\right]$ across all possible misalignment angles, where $\mathrm{E}[x]$ denotes the expectation of the variable $x$. The best $\Phi_{b}$ values that can achieve the maximum value of $\mathrm{E}\left[\bar{\rho}_{\max }\right]$ are shown in Table 1 . It can be seen that mirror-aided non-LOS configuration requires smaller $\Phi_{b}$ than LOS configuration to achieve the best overall performance.

Table 1. Best auxiliary semi-angle to achieve the maximum of expected value of ASE

\begin{tabular}{|l|c|c|c|c|}
\hline Scheme and Option & \multicolumn{2}{|c|}{ LOS } & \multicolumn{2}{c|}{ Mirror-aided non-LOS } \\
\hline & $\Phi_{b}$ & $\mathrm{E}\left[\bar{\rho}_{\max }\right](\mathrm{bit} / \mathrm{s} / \mathrm{Hz} / \mathrm{cell})$ & $\Phi_{b}$ & $\mathrm{E}\left[\bar{\rho}_{\max }\right](\mathrm{bit} / \mathrm{s} / \mathrm{Hz} / \mathrm{cell})$ \\
\hline FR & $13^{\circ}$ & 2.8 & $5^{\circ}$ & 0.9 \\
\hline IB 1, option 1 & $14^{\circ}$ & 0.9 & $8^{\circ}$ & 0.9 \\
\hline IB 1, option 2 & $13^{\circ}$ & 1.3 & $8^{\circ}$ & 1.3 \\
\hline IB 2. option 1 & $13^{\circ}$ & 0.4 & $8^{\circ}$ & 0.4 \\
\hline IB 2, option 2 & $12^{\circ}$ & 0.6 & $8^{\circ}$ & 0.6 \\
\hline
\end{tabular}

\section{CONCLUSIONS}

Two-tier optical attocell networks using different backhaul link configurations have different performance at misalignment scenarios. The impact of misalignment varies with horizontal misalignment angle and auxiliary semi-angle. By comparing the simulation results of two backhaul configurations and different frequency reuse schemes, we found that networks using LOS backhaul configuration transition from noise-limited regime to interference-limited regime when the misalignment angle and the semi-angle of auxiliary LEDs become larger. Networks using mirror-aided non-LOS backhaul configuration are always in the interference-limited regime. FR scheme provide the best overall performance for networks in the noise-limited regime. Networks using IB scheme can achieve better performance in the interference-limited regime. It is also found that LOS backhaul configuration is more vulnerable to misalignment than mirror-aided non-LOS configuration.

\section{REFERENCES}

[1] H. Kazemi, M. Safari, and H. Haas, "A wireless optical backhaul solution for optical attocell networks", IEEE Transactions on Wireless Communications, vol. 18, pp. 807-823, 2019.

[2] Y. Wu, P. Audenaert, M. Pickavet, and D. Colle, "Mirror-aided non-LOS VLC channel characterizations with a time-efficient simulation model", Photonic Network Communications, vol. 38, pp. 151-166, 2019.

[3] J. Armstrong and B. J. C. Schmidt, "Comparison of asymmetrically clipped optical OFDM and DC-biased optical OFDM in AWGN", IEEE Communications Letters, vol. 12, pp. 343-345, 2008.

[4] J. R. Barry, J. M. Kahn, W. J. Krause, E. A. Lee, and D. G. Messerschmitt, "Simulation of multipath impulse response for indoor wireless optical channels", IEEE Journal on Selected Areas in Communications, vol. 11, pp. 367-379, 1993. 